\title{
On an Abstract Differential Equation and Its Application to Positive Eigenvalues of Schrödinger Operators
}

\author{
By
}

M. W. WONG*

\begin{abstract}
A second order differential equation in Hilbert space is shown to have only trivial solutions. This functional analytic result is then used to derive an upper bound for the set of positive eigenvalues of the one-body Schrödinger operator.
\end{abstract}

\section{§1. Introduction}

The theory of the Schrödinger operator $-\Delta+q(x)$, where $\Delta$ is the Laplacian and $q(x)$ a measurable function on $\boldsymbol{R}^{n}$, initiated by Kato in [7], has become a highly developed discipline in mathematical analysis. The spectral theory of the Schrödinger operator is by now fairly well understood.

In [12], Wigner and von Neumann have constructed a function $q(x)$ such that $-\Delta+q$ has the positive number 1 as an eigenvalue. This phenomenon suggests that a natural spectral problem for the operator $-\Delta+q(x)$ is to give a good upper bound for the set of positive eigenvalues under reasonable assumptions on $q(x)$ in a neighbourhood of infinity. This problem has been studied by Agmon [1, 2], Kato [8], Odeh [9] and Simon [11] among others. An exposition of the contributions by these authors can be found in Reed and Simon [10], and most recently, Eastham and Kalf [5]. A discussion on the hypotheses and conclusions of the papers of Agmon and Simon can be found in Jansen and Kalf [6]. Eastham [4] has obtained an upper bound for the set of positive eigenvalues of the Sturm-Liouville operator $-d^{2} / d x^{2}+q(x)$ on $(0, \infty)$.

The object of this paper is to combine the techniques of Eastham in [4] and Jansen and Kalf in [6] to construct a second order differential equation in Hilbert space with only trivial solutions. The details are given in Sections 2 and 3. As an application, we derive an upper bound (similar to that of Eastham) for the set of positive eigenvalues of the Schrödinger operator $-\Delta+q(x)$. For technical reasons, we assume that the function $q(x)$ is so smooth that the regularity and

Communicated by S. Matsuura, December 13, 1982.

* Department of Mathematics, York University, 4700 Keele St., Downsview, Ontario, Canada, M3J 1P3. 
unique continuation properties for the solutions of elliptic partial differential equations can be applied. Detailed assumptions on $q(x)$ are given in Section 4 . Since the proofs are quite complicated, an attempt is made to supply with full details. In this respect the paper is sufficiently selfcontained.

Acknowledgements are made to Professor F. V. Atkinson for suggesting the topic, Professor T. Kato for pointing out several mistakes and unrealistic assumptions in the first version of this paper, and the referee for the detailed comments and suggestions which have led to this revised version.

\section{§2. An Abstract Differential Equation}

Let $\mathscr{H}$ be a complex Hilbert space with inner product and norm denoted by $\langle$,$\rangle and \|\|$ respectively. Denote by $L^{2}(\mathscr{K})$ the space of all functions

$$
u:(0, \infty) \longrightarrow \mathscr{H}
$$

such that $u(t)$ is strongly measurable on $(0, \infty)$ and

$$
\int_{0}^{\infty}\|u(t)\|^{2} d t<\infty
$$

We denote by $C^{k}(\mathscr{H}), 1 \leqq k<\infty$, the space of all functions

$$
u:(0, \infty) \longrightarrow \mathscr{H}
$$

such that its first $k$ strong derivatives are strongly continuous on $(0, \infty)$.

Let $B(\mathscr{K})$ denote the set of all bounded linear operators on $\mathscr{H}$. With the usual definition of operator norm, $B(\mathscr{H})$ is a Banach space. The derivative of a function

$$
T:(0, \infty) \longrightarrow B(\mathscr{H})
$$

will also be taken in the strong sense.

Let $A$ be a nonnegative, unbounded linear operator with domain $\mathscr{D}(A)$ dense in $\mathscr{H}$. Let $T_{0}(t)$ and $T_{1}(t)$ be self-adjoint bounded linear operators defined on $\mathscr{H}$ satisfying the following conditions :

(i) The derivative $T_{0}^{\prime}(t)$ of $T_{0}(t)$ exists and is a self-adjoint bounded linear operator on $\mathscr{H}$ for $t$ large enough, say for $t \geqq \xi_{1}$.

(ii) For any $\varepsilon>0$, there is a $\xi_{2}>0$ such that for all functions $u:(0, \infty) \rightarrow$ $\mathscr{H}$ and $v:(0, \infty) \rightarrow \mathscr{H}$, we have

$$
\begin{aligned}
& \left|\left\langle T_{0} u, v\right\rangle\right| \leqq \varepsilon\|u\|\|v\|, \\
& \left|\left\langle T_{1} u, v\right\rangle\right| \leqq \varepsilon\|u\|\|v\|,
\end{aligned}
$$

whenever $t \geqq \xi_{2}$.

(iii) There exist nonnegative constants $L$ and $K$ such that for any $\varepsilon>0$, there is a $\xi_{3}>0$ such that for all $u, v$ as in (ii), 


$$
\begin{gathered}
\left\langle t T_{0}^{\prime} u, u\right\rangle \leqq(L+\varepsilon)\|u\|^{2} \\
\left|\left\langle t T_{1} u, v\right\rangle\right| \leqq(K+\varepsilon)\|u\|\|v\|
\end{gathered}
$$

whenever $t \geqq \hat{亏}_{3}$.

The abstract differential equation to be studied is of the following form:

$$
v^{\prime \prime}(t)+\left\{\lambda-\left(t^{-2} A+T_{0}(t)+T_{1}(t)\right)\right\} v(t)=0, \quad 0<t<.=0 .
$$

where $\lambda$ is a real number.

We assume that (2.5) satisfies the following unique continuation property: (U.C.P.) If $v(t) \in C^{2}(\mathscr{H}) \cap L^{2}(\mathscr{H})$ is a solution of (2.5) which is not identically zero, then there is a sequence $\left\{t_{n}\right\}$ of real numbers such that

$$
\lim _{n \rightarrow \infty} t_{n}=\infty
$$

and

$$
\left\|v\left(t_{n}\right)\right\|>0, n=1,2, \cdots .
$$

Theorem 2.1. The abstract differential equation (2.5) has no nunzero solutions in $L^{2}(\mathscr{R}) \cap C^{2}(\mathscr{H})$ if

$$
\lambda>\frac{1}{2}\left\{K^{2}+L+K \sqrt{ }\left(2 L+K^{2}\right)\right\} .
$$

Proof. We assume $K>0$ and $L>0$. Let $v \in C^{2}(\mathscr{H}), L^{2}$ it be a nonzero solution of (2.5). Define

$$
h(t)=\left\|v^{\prime}(t)\right\|^{2}+\left\langle\left(\lambda-t^{-2} A-T_{0}(t)\right) v(t), v(t)\right\rangle, \quad t>\max \left(\xi_{1}, \ldots\right.
$$

Differentiating and applying (2.5), then for $t>\max \left(\xi_{1}, \xi_{2}, \xi_{3}\right)$.

$$
h^{\prime}(t)=-\left\langle T_{0}^{\prime}(t) v(t), v(t)\right\rangle+2 \operatorname{Re}\left\langle T_{1}(t) v(t), v^{\prime}(t)\right\rangle+2 t^{-3 \prime} A v^{\prime} t^{\prime} . \imath t^{\prime} \cdot .
$$

By (2.3) and (2.4), the following inequality is valid:

$$
h^{\prime}(t) \geqq-t^{-1}\left\{2 K_{1}\|v(t)\|\left\|v^{\prime}(t)\right\|+L_{1}\|v(t)\|^{2}-2 t^{-2}\left\langle\cdot t v(t), v^{\prime} t \quad\right.\right.
$$

where $K_{1}$ and $L_{1}$ lie within an arbitrary but fixed $\varepsilon$ of $K$ and $L$ respectively and it is understood that (2.8) is valid for $t$ large enough.

Let $c \in(-1,1)$. Then

$$
\begin{aligned}
2^{2.9)} & \left.-\left\{c t^{-1} \operatorname{Re} / v(t), v^{\prime}(t)\right\rangle\right\}^{\prime} \\
& \left.=-\left\{c t^{-1} \operatorname{Re}\left\langle v(t), v^{\prime \prime}(t)\right\rangle+c t^{-1} \operatorname{Re}{ }^{\prime} v^{\prime}(t), v^{\prime}(t)\right\rangle-c t^{-2} \operatorname{Re} v^{\prime} v^{\prime}(t), \imath^{\prime} t^{\prime}\right\} \\
& \left.\left.=-\left\{c t^{-1}\left\|v^{\prime}(t)\right\|^{2}-c t^{-1 /} \imath^{\prime}(t),\left(\lambda-t^{-2} A-T_{0}(t)-T_{1}(t)\right) v(t)\right\rangle-c t^{-2} \operatorname{Re} v^{\prime}(t), v^{\prime}(t)\right\rangle\right\} .
\end{aligned}
$$

Adding (2.8) and (2.9), letting $\alpha>0$, we get

$$
\begin{aligned}
& \left\{h^{\prime}(t)-c t^{-1} \operatorname{Re}\left\langle v(t), v^{\prime}(t)\right\rangle\right\}^{\prime} \\
\geqq & -t^{-1}\left\{c\left\|v^{\prime}\right\|^{2}+2 K_{2}\|v\|\left\|v^{\prime}\right\|+\left(L_{2}-c \lambda\right)\|v\|^{2}+(c-2) t^{-2,} \text {. A . . . }\right\}
\end{aligned}
$$




$$
\geqq-t^{-1}\left\{\left(c+\alpha K_{2}\right)\left\|v^{\prime}\right\|^{2}+\left(L_{2}-c \lambda+\alpha^{-1} K_{2}\right)\|v\|^{2}+(c-2) t^{-2}\langle A v, v\rangle\right\}
$$

where $K_{2}$ and $L_{2}$ are constants lying within $\varepsilon$ of $K$ and $L$ respectively and it is understood that (2.10) is valid for $t$ large enough.

By (2.6) and (2.10), it follows that for $t$ large enough, say for $t \geqq T=T(\varepsilon)$,

$$
\left\{h(t)-c t^{-1} \operatorname{Re}\left\langle v(t), v^{\prime}(t)\right\rangle\right\}^{\prime} \geqq-\gamma t^{-1}\left\{h(t)-c t^{-1} \operatorname{Re}\left\langle v(t), v^{\prime}(t)\right\rangle\right\}
$$

provided that

$$
\gamma>c+\alpha K_{3} ; \lambda \gamma>L_{3}-c \lambda+\alpha^{-1} K_{3} ; 2-c>\gamma ;
$$

where $K_{3}$ and $L_{3}$ are constants lying within $\varepsilon$ of $K$ and $L$ respectively.

(2.11) is ralid with $0<\gamma<1$ provided that

$$
(1-c)>\alpha K ; \lambda-L+c \lambda>\alpha^{-1} K \text {. }
$$

The inequalities in (2.12) are valid for some $\alpha>0$ if

$$
(1-c)(\lambda-L+c \lambda)>K^{2}
$$

Using (2.13), the inequalities in (2.12) are valid for some $\alpha>0$ if

$$
\lambda>K^{2}\left(1-c^{2}\right)^{-1}+L(1+c)^{-1} .
$$

Considering $\hbar^{-2}\left(1-c^{2}\right)^{-1}+L(1+c)^{-1}$ as a function of $c \cong(-1,1)$ the minimum value is

$$
\frac{1}{2}\left\{K^{2}+L+K \sqrt{\left(2 L+K^{2}\right)}\right\}
$$

and is attained at

$$
c_{0}=L^{-1}\left\{K^{2}+L-K \sqrt{ }\left(2 L+K^{2}\right)\right\} .
$$

Hence by $(2.11), \cdots,(2.15)$ and $(2.16)$, we have proved that there is a $\gamma \equiv(0,1)$ such that

$$
\left\{h(t)-c_{n} t^{-1} \operatorname{Re}\left\langle v(t), v^{\prime}(t)\right\rangle\right\}^{\prime} \geqq-\gamma t^{-1}\left\{h(t)-c_{0} t^{-1} \operatorname{Re}\left\langle v(t), v^{\prime}(t)\right\rangle\right\}
$$

for $t \geqq T=T(\varepsilon)$, provided that

$$
\lambda>\frac{1}{2}\left\{K^{2}+L+K \sqrt{ }\left(2 L+K^{2}\right)\right\} .
$$

CLAIM: There is an $\eta \in(0, \infty)$ such that

$$
h(t)-c_{0} t^{-1} \operatorname{Re}\left\langle v(t), v^{\prime}(t)\right\rangle>0, \quad t \geqq \eta_{1} .
$$

The proof of $(2.18$, will be given in Section 3 .

Let $\tau=\max (\tau, T)$. Then (2.17) and (2.18) imply that 


$$
\int_{=}^{t} \begin{aligned}
& \left\{h(s)-c_{0} s^{-1} \operatorname{Re}\left\langle v(s), v^{\prime}(s)\right\rangle\right\}^{\prime} \\
& \quad h(s)-c_{0} s^{-1} \operatorname{Re}\left\langle v(s), v^{\prime}(s)\right\rangle
\end{aligned} d s \geqq-\gamma \int_{\tau}^{t} \frac{d s}{s}
$$

Hence

$$
h(t)-c_{0} t^{-1} \operatorname{Re}\left\langle v(t), v^{\prime}(t)\right\rangle \geqq c^{\prime} t^{-\gamma}, \quad t>\tau
$$

where $c^{\prime}$ is a positive constant.

Recalling the definition of $h(t)$, we have

$$
\begin{aligned}
& \left.\left\|v^{\prime}(t)\right\|^{2}+\left\langle\left(\lambda-t^{-2} A-T_{0}(t)\right) v(t), v(t)\right\rangle-c_{0} t^{-1} \operatorname{Re}\left\langle v^{\prime}(t), v^{\prime}, t^{\prime}\right\rangle\right\rangle \\
& \geqq c^{\prime} t^{-\ddot{ }}, \quad t>\tau .
\end{aligned}
$$

By (2.1),

$$
\left\|v^{\prime}(t)\right\|^{2}+\left\langle\left(\lambda_{1}-\frac{1}{2} t^{-2}-4\right) v(t), v(t)\right\rangle \geqq C_{1} t^{-r}
$$

where $C_{1}$ and $\lambda_{1}$ are positive constants and (2.21) holds for $t$ large enough, say for $t \geqq \sigma>\tau$.

Since $v(t) \in L^{2}(\mathscr{H})$, we have

$$
\int_{0}^{\infty}\|v(t)\|^{2} d t<\infty
$$

Hence the function $\|v(t)\|^{2}$ on $(0, \infty)$ cannot be monotone increasing on any interval of the form $(\beta, \infty), \beta \geqq 0$.

Since

$$
\frac{d}{d t}\left\|v^{\prime}(t)\right\|^{2}=2 \operatorname{Re}\left\langle v(t), v^{\prime}(t)\right\rangle
$$

it follows that there exists a sequence of real numbers $\left\{t_{n}\right\}, t_{2} \geqq \sigma$, such that

$$
\lim _{n \rightarrow \infty} t_{n}=\infty
$$

and

$$
\operatorname{Re}\left\langle v\left(t_{n}\right), v^{\prime}\left(t_{n}\right)\right\rangle \leqq 0
$$

Hence by (2.5), we get

$$
\begin{aligned}
& -\operatorname{Re}\left\langle v\left(t_{n}\right), v^{\prime}\left(t_{n}\right)\right\rangle+\operatorname{Re}\left\langle v(\sigma), v^{\prime}(\sigma)\right\rangle \\
& =-\int_{\sigma}^{t_{n}} \frac{d}{d t}\left\{\operatorname{Re}\left\langle v(t), v^{\prime}(t)\right\rangle\right\} d t \\
& =-\int_{\sigma}^{t_{n}}\left\{\left\|v^{\prime}(t)\right\|^{2}+\operatorname{Re}\left\langle v(t), v^{\prime \prime}(t)\right\rangle\right\} d t \\
& =-\int_{\sigma}^{t_{n}}\left\|v^{\prime}(t)\right\|^{2} d t+\int_{\sigma}^{t_{n}} \operatorname{Re}\left\langle v^{\prime}(t),\left(\lambda-t^{-2}-A-T_{0}(t)-T_{3}\left(t^{\prime}\right) v^{\prime}(t)\right\rangle d t .\right.
\end{aligned}
$$

Hence by (2.1) and (2.23), we get 


$$
-\int_{\sigma}^{t_{n}}{ }_{1} v^{\prime}(t) \|^{2} d t+\int_{\sigma}^{t_{n}}\left\langle v(t),\left(\lambda_{1}-t^{-2} A\right) v(t)\right\rangle d t \geqq \operatorname{Re}\left\langle v(\sigma), v^{\prime}(\sigma)\right\rangle .
$$

Integrating $(2.21$, we get

$$
\int_{\sigma}^{t_{n}}\left\|\imath^{\prime}(t)\right\|^{2} d t+\int_{\sigma}^{t_{n}}\left\langle v(t),\left(\lambda_{1}-\frac{1}{2} t^{-2} A\right) v(t)\right\rangle d t \geqq C_{2} t_{n}^{1-\gamma}-C_{2} \sigma^{1--}
$$

where $C_{2}$ is a positive constant.

Hence by (2.24) and (2.25), we have

$$
\int_{\sigma}^{t_{n}}\left\langle v^{\prime}(t),\left(2 \lambda_{1}-\frac{3}{2} t^{-2} A\right) v(t)\right\rangle d t \geqq C_{2} t_{n}^{1-\gamma}-C_{2} \sigma^{1-\gamma}+\operatorname{Re}\left\langle v(\sigma), v^{\prime}(\sigma)\right\rangle .
$$

Using $0<\gamma<1$ and (2.22), we get

$$
\lim _{n \rightarrow \infty} \int_{\sigma}^{t_{n}}\left\langle v(t),\left(2 \lambda_{1}-\frac{3}{2} t^{-2} A\right) v(t)\right\rangle d t=\infty .
$$

Since $A$ is a nonnegative operator, (2.26) implies that

$$
\lim _{n \rightarrow \infty} \int_{\sigma}^{t_{n}}\|v(t)\|^{2} d t=c 0 .
$$

But (2.27) contradicts the fact that $v \in L^{2}(\mathscr{H})$. This proves the theorem.

Remark. By slightly modifying the proof of the theorem, the cases when $I=0$ or $L=0$ can be covered. We omit the details.

\section{\$3. Continuation of the Proof of Theorem 2.1}

In this section we prove the claim in (2.18).

Proposition 3.1. There exists an $\eta \in(0, \infty)$ such that

$$
\left\|v^{\prime}(t)\right\|^{\prime 2} \div\left\langle\left(\lambda-t^{-2} A-T_{0}(t)\right) v(t), v(t)\right\rangle-c_{0} t^{-1} \operatorname{Re}\left\langle v(t), v^{\prime}(t),>0\right.
$$

for $t \geqq \eta$, provided that

$$
\lambda>\frac{1}{2}\left\{K^{2}+L+K \sqrt{ }\left(\overline{2} \bar{L}+\bar{K}^{2}\right)\right\} .
$$

Proof. For $t \geqq T=T(\varepsilon)$, define

$$
F(t)=i^{\prime \prime} r^{\prime}(t) \|^{\prime \prime 2}+\left\langle\left(\lambda-t^{-2} A-T_{0}(t)\right) v(t), v(t)\right\rangle+(1-d) t^{-1} \operatorname{Re}\left\langle v^{\prime}(t), v^{\prime}(t),\right.
$$

where $d=1+c_{0}$. Then using (2.5),

$$
\begin{aligned}
(t F(t))^{\prime}= & (2-d)\left\|v^{\prime}\right\|^{2}+(2-d) t^{-2}\langle A v, v\rangle+2 \operatorname{Re}\left\langle t T_{1} v, v^{\prime},\right. \\
& +\left(\left\{d\left(\lambda-T_{0}\right)-t T_{0}^{\prime}+(1-d) T_{1}\right\} v, v\right\rangle \\
\geqq & (2-d)\left\|v^{\prime}\right\|^{2}+2 \operatorname{Re}\left\langle t T_{1} v, v^{\prime}\right\rangle+\left\langle\left\{d\left(\lambda-T_{0}\right)-t T_{0}^{\prime}+(1-d) T_{1}\right\} v^{\prime}, v^{\prime}, .\right.
\end{aligned}
$$

We have used the nonnegativity of $A$ to obtain the above inequality. Let $\varepsilon>0$. 
Then for $t$ large enough, we have

$$
(t F(t))^{\prime} \geqq(2-d)\left\|v^{\prime}\right\|^{2}-2(K+\varepsilon)\|v\|\left\|v^{\prime}\right\|+\{d \lambda-L-(d+2) \varepsilon\}\|v\|^{2} .
$$

The discriminant of the quadratic expression

$$
(2-d)\left\|v^{\prime}\right\|^{2}-2(K+\varepsilon)\|v\|\left\|v^{\prime}\right\|+\{d \lambda-L-(d+2) \varepsilon\}\|v\|^{2}
$$

is given by

$$
\begin{aligned}
& 4(K+\varepsilon)^{2}-4(2-d)\{d \lambda-L-(d+2) \varepsilon\} \\
= & 4\left\{K^{2}-\left(1-c_{0}^{2}\right) \lambda+\left(1-c_{0}\right) L+f(\varepsilon)\right\}
\end{aligned}
$$

where

$$
f(\varepsilon)=2 K \varepsilon+\varepsilon^{2}+\left(1-c_{0}\right)\left(3+c_{0}\right) \varepsilon .
$$

Since

$$
\lambda>\frac{1}{2}\left\{h^{2}+L+K \sqrt{ }\left(2 \bar{L}+\bar{h}^{2}\right)\right\},
$$

we can choose $s$ small enough so that

$$
\begin{aligned}
& 4\left\{K^{2}-\left(1-c_{0}^{2}\right) \lambda+\left(1-c_{0}\right) L+f(\varepsilon)\right\} \\
< & \left.4\left\{K^{2}-\frac{1}{2}\left(1-c_{0}^{2}\right)\left[K^{2}+L+K \sqrt{(2 L} \bar{\mp} \bar{K}^{2}\right)\right]+\left(1-c_{0}\right) L\right\}=0 .
\end{aligned}
$$

Hence for $t$ large enough,

$$
(t F(t))^{\prime} \geqq 0 .
$$

Let $t \geqq 0, m \geqq 0$ and $\rho>0$. Following Jansen and Kalf [4], we define

$$
F(m, \rho, t)=\left\|v_{m}^{\prime}\right\|^{2}-t^{-2}\left\langle v_{m}^{\prime}, d v_{m}\right\rangle+\left\langle\left\{\lambda-T_{0}-\rho t^{-1}+t^{-2} m(m+1)\right\} v_{m}, v_{m}\right\rangle
$$

where

$$
v_{m}=t^{m} \imath^{\prime}
$$

Using (2.5), we get

$$
v_{m}^{\prime \prime}=2 m t^{-1} v_{m}^{\prime}-m(m+1) t^{-2} v_{m}+t^{-2} A v_{m}-\left(\lambda-T_{0}-T_{1}\right) v_{m} .
$$

Hence

$$
\begin{aligned}
\left\{t^{2} F(m, \rho, t)\right\}^{\prime}= & t\left\{2(2 m+1)\left\|v_{m}^{\prime}\right\|^{2}+2 \operatorname{Re}\left\langle\left(t T_{1}-\rho\right) v_{m}, v_{m}^{\prime}\right\rangle\right. \\
& \left.+\left\langle\left[2\left(\lambda-T_{0}\right)-t T_{0}^{\prime}-\rho t^{-1}\right] v_{m}, v_{m}\right\rangle\right\} .
\end{aligned}
$$

Let $s>0$. Then for $t$ large enough,

$$
\left\{t^{2} F(m, \rho, t)\right\}^{\prime} \geqq t\left\{2(2 m+1)\left\|v_{m}^{\prime}\right\|^{2}-2(K+\varepsilon+\rho)\left\|v_{m}\right\|\left\|v_{m}^{\prime}\right\|+(2 \lambda-L-3 \varepsilon-\rho \varepsilon)\left\|v_{m}\right\|^{2}\right\} .
$$

Choose a fixed $\rho$ such that

$$
\left.(K+\rho)^{2}-2\left\{K^{2}+L+K \sqrt{(2 L+} K^{2}\right)\right\}+2 L<0 .
$$

Hence for $\varepsilon$ small enough, 
$(K+\varepsilon+\rho)^{2}-2(2 m+1)(2 \lambda-L-3 \varepsilon-\rho \varepsilon) \leqq(K+\varepsilon+\rho)^{2}-2(2 \lambda-L-3 \varepsilon-\rho \varepsilon)<0$.

Hence there is a $t^{\prime}>0$ such that

$$
\left\{t^{2} F(m, \rho, t)\right\}^{\prime} \geqq 0, \quad t \geqq t^{\prime}, m \geqq 0 .
$$

By (U.C.P.), let $t_{1} \geqq t^{\prime}$ be such that

$$
\left\|v\left(t_{1}\right)\right\|>0 .
$$

Then

$$
\begin{aligned}
& t_{1}^{2-2 m} F\left(m, \rho, t_{1}\right) \\
\geqq & -\left\langle v\left(t_{1}\right), A v\left(t_{1}\right)\right\rangle+m(m+1)\left\|v\left(t_{1}\right)\right\|^{2}+\left\langle t_{1}^{2}\left(\lambda-T_{0}\left(t_{1}\right)-\rho t_{1}^{-1}\right) v\left(t_{1}\right), v\left(t_{1}\right)\right\rangle .
\end{aligned}
$$

Let $m_{1}>(1-d) / 2$ be such that

$$
t_{1}^{2-2 m_{1}} F\left(m_{1}, \rho, t_{1}\right)>0 .
$$

Hence

$$
t_{1}^{2} F\left(m_{1}, \rho, t_{1}\right)>0 .
$$

Then by (3.2) and (3.3), we have

$$
t^{2} F\left(m_{1}, \rho, t\right) \geqq t_{1}^{2} F\left(m_{1}, \rho, t_{1}\right)>0, \quad t \geqq t_{1} .
$$

Hence

$$
t^{-2 m_{1}} F\left(m_{1}, \rho, t\right)>0, \quad t \geqq t_{1} .
$$

But

$$
\begin{aligned}
& t^{-2 m_{1}} F\left(m_{1}, \rho, t\right) \\
= & t^{-2 m_{1}}\left\{\left\|v_{m_{1}}^{\prime}\right\|^{2}-t^{-2}\left\langle v_{m_{1}}, A v_{m_{1}}\right\rangle+\left\langle\left[\lambda-T_{0}-\rho t^{-1}+t^{-2} m_{1}\left(m_{1}+1\right)\right] v_{m_{1}}, v_{m_{1}}\right\rangle\right\} \\
= & \left\|v^{\prime}\right\|^{2}+2 m_{1} t^{-1} \operatorname{Re}\left\langle v^{\prime}, v\right\rangle-t^{-2}\langle v, A v\rangle+\left\langle\left(\lambda-T_{0}\right) v, v\right\rangle+\left(2 m_{1}+1\right) m_{1} t^{-2}\|v\|^{2}-\rho t^{-1}\|v\|^{2} \\
\leqq & \left\|v^{\prime}\right\|^{2}+2 m_{1} t^{-1} \operatorname{Re}\left\langle v^{\prime}, v\right\rangle-t^{-2}\langle v, A v\rangle+\left\langle\left(\lambda-T_{0}\right) v, v\right\rangle
\end{aligned}
$$

provided that we choose $t$ so large that

$$
\left(2 m_{1}+1\right) m_{1} t^{-1}<\rho .
$$

Hence there is a $t_{2}>t_{1}$ such that for $t \geqq t_{2}$,

$$
\begin{aligned}
& t^{-2 m_{1}} F\left(m_{1}, \rho, t\right) \\
\leqq & \left\|v^{\prime}\right\|^{2}+\left\langle\left(\lambda-t^{-2} A-T_{0}\right) v, v\right\rangle+(1-d) t^{-1} \operatorname{Re}\left\langle v^{\prime}, v\right\rangle+\left(d+2 m_{1}-1\right) t^{-1} \operatorname{Re}\left\langle v^{\prime}, v\right\rangle \\
= & F(t)+\left(d+2 m_{1}-1\right) t^{-1} \operatorname{Re}\left\langle v^{\prime}, v\right\rangle .
\end{aligned}
$$

Since $v \in L^{2}(\mathscr{H}),\|v\|^{2}$ cannot be monotone increasing on any interval of the form $(\beta, \infty), \beta \geqq 0$. Hence there is a $t_{3} \geqq t_{2}$ such that 


$$
\operatorname{Re}\left\langle v^{\prime}\left(t_{3}\right), v\left(t_{3}\right)\right\rangle \leqq 0
$$

By (3.4) and (3.5).

$$
0<t_{3}^{-2 m_{1}} F\left(m_{1}, \rho, t_{3}\right) \leqq F\left(t_{3}\right)
$$

Hence

$$
t_{3} F\left(t_{3}\right)>0 \text {. }
$$

By (3.1) and (3.6), we have

$$
t F(t)>0
$$

for $t$ large enough. This proves the proposition.

\section{$\S 4$. The Positive Eigenvalues}

In this section we use Theorem 2.1 to study the positive eigenvalues of the Schrödinger operator.

Theorem 4.1. Let $q \in L_{\mathrm{loc}}^{2}\left(\boldsymbol{R}^{n}\right), n \geqq 2$, be a real-valued function satisfying the following conditions:

(1) $q(x)$ is locally Hölder continuous on $\boldsymbol{R}^{n}$.

(2) $q(x)=q_{0}(x)+q_{1}(x)$, where $q_{0}(x)$ and $q_{1}(x)$ are real-valued continuous functions defined on $\boldsymbol{R}^{n}$.

(3) $\lim _{|x| \rightarrow \infty} q_{0}(x)=0$ and $\lim _{|x| \rightarrow \infty} q_{1}(x)=0$.

(4) The radial derivative $q_{0}^{\prime}(x)$ of $q_{0}(x)$ exists.

(5) $\limsup _{|x| \rightarrow \infty}|x|\left|q_{1}(x)\right|=K, \quad K<\infty$.

(6) $\limsup _{|x| \rightarrow \infty}|x| q_{0}^{\prime}(x)=L, \quad 0 \leqq L<\infty$.

Let $P$ be a self-adjoint extension of the operator

$$
-\lrcorner+q: C_{0}^{\infty}\left(\boldsymbol{R}^{n}\right) \longrightarrow L^{2}\left(\boldsymbol{R}^{n}\right) .
$$

Then $P$ has no eigenualues in $(\Lambda, \infty)$, where

$$
\Lambda=\frac{1}{2}\left\{K^{2}+L+K \sqrt{ }\left(2 L+K^{2}\right)\right\} .
$$

Remark. Following Agmon [1,2], Jansen and Kalf [6] and Simon [11], we have assumed that the function $q(x)$ is fairly smooth. The smoothness conditions on $q(x)$ need only be imposed in a neighbourhood of infinity.

Proof. We first consider the case when $n \geqq 3$. Let $\lambda>\Lambda$ be an eigenvalue. Let $u$ be a corresponding eigenfunction. By hypothesis (1), $u$ can be assumed to be in $C^{2}\left(\boldsymbol{R}^{n}\right)$. Introducing polar coordinates $t=|x|, \xi=t^{-1} x$; writing $v(t, \tilde{\xi})$ $=t^{(n-1) / 2} u(t, \xi)$ and letting

$$
S^{n-1}=\left\{x \in \boldsymbol{R}^{n}:|x|=1\right\},
$$


it follows that the function

$$
(0, \infty) \ni t \longmapsto v(t, \cdot) \in L^{2}\left(S^{n-1}\right)
$$

is in $L^{2}(\mathscr{K}) \cap C^{2}(\mathscr{H})$, where $\mathscr{H}=L^{2}\left(S^{n-1}\right) . \quad \mathscr{H}$ is a Hilbert space with inner product

$$
\langle f, g\rangle=\int_{S^{n-1}} f(\xi) \overline{g(\xi)} d \xi, \quad f, g \in L^{2}\left(S^{n-1}\right) .
$$

We also have

$$
v^{\prime \prime}+\left\{\lambda-t^{-2}(A+\mu)-q_{0}-q_{1}\right\} v=0, \quad 0<t<\infty
$$

where $\mu=\frac{1}{4}(n-1)(n-3) ; A$ is the negative of the Laplace Beltrami operator and is hence a nonnegative, unbounded linear operator on $L^{2}\left(S^{n-1}\right)$.

Define operators $T_{0}(t)$ and $T_{1}(t)$ on $\mathscr{H}$ for $t>0$ by

$$
\left(T_{0}(t) f\right)(\xi)=q_{0}(t, \xi) f(\xi), \quad f \in \mathscr{H}
$$

and

$$
\left(T_{1}(t) f\right)(\xi)=q_{1}(t, \xi) f(\xi), \quad f \in \mathscr{H} .
$$

Using the real-valuedness, continuity and hypothesis (3) of $q_{0}$ and $q_{1}$, it follows that $T_{0}(t)$ and $T_{1}(t)$ are self-adjoint bounded linear operators on $\mathscr{H}$ for $t>0$. Using (4.3) and hypothesis (6), for $t$ large enough,

$$
\left(T_{0}^{\prime}(t) f\right)(\xi)=q_{0}^{\prime}(t, \xi) f(\xi), \quad f \in \mathscr{K}
$$

and is a self-adjoint bounded linear operator on $\mathcal{H}$. Let $\varepsilon>0$. Then there is a $t_{1}>0$ such that for all functions $\alpha:(0, \infty) \rightarrow \mathscr{H}$ and $\beta:(0, \infty) \rightarrow \mathscr{K}$,

$$
\begin{gathered}
\left|\left\langle T_{0} \alpha, \beta\right\rangle\right| \leqq \varepsilon\|\alpha\|\|\beta\|, \\
\left|\left\langle T_{1} \alpha, \beta\right\rangle\right| \leqq \varepsilon\|\alpha\|\|\beta\|, \\
\left\langle t T_{0}^{\prime} \alpha, \alpha\right\rangle \leqq(L+\varepsilon)\|\alpha\|^{2}, \\
\left|\left\langle t T_{1} \alpha, \beta\right\rangle\right| \leqq(K+\varepsilon)\|\alpha\|\|\beta\|,
\end{gathered}
$$

whenever $t \geqq t_{1}$.

We have used hypotheses (3), (5) and (6) to obtain the above inequalities. Rewrite (4.2) in the form

$$
v^{\prime \prime}+\left\{\lambda-t^{-2}(A+\mu)-T_{0}(t)-T_{1}(t)\right\} v=0, \quad 0<t<\infty .
$$

The unique continuation property for the operator

$$
-\Delta+q-\lambda
$$

(see, for example, Aronszajn [3]), implies that the abstract differential equation (4.10) satisfies the (U.C.P.) property formulated in Section 2. Hence by The- 
orem 2.1 ,

$$
v(t, \cdot) \notin L^{2}(\mathscr{H}) \cap C^{2}(\mathscr{H}) .
$$

This is a contradiction. Hence $P$ has no eigenvalues in $(\Lambda, \infty)$.

For the case when $n=2$, the above proof can be modified by writing (4.2) in the form

where

$$
\imath^{\prime \prime}+\left\{\lambda-t^{-2} A-q_{0}-\tilde{q}_{1}\right\} v=0, \quad 0<t<\infty
$$

$$
\tilde{q}_{1}(t, \xi)=q_{1}(t, \tilde{\xi})-\frac{1}{4} t^{-2}
$$

and replacing $T_{1}(t)$ by $\tilde{T}_{1}(t)$ where

$$
\left(\widetilde{T}_{1}(t) f\right)(\xi)=\tilde{q}_{1}(t, \xi) f(\xi), \quad \int \in \mathscr{H} .
$$

This completes the proof of the theorem.

\section{References}

[1- Agmon, S., Lower bounds for solutions of Schrödinger-type equations in unbounded domains, Proc. Inter. Conf. Functional Anal. Related Topics, Tokyo (1969), 216-224.

[2- — Lower bounds for solutions of Schrödinger equations, J. Analyse Math. 23 (1970), 1-25.

[3- Aronszajn, N., A unique continuation theorem for solutions of elliptic partial differential equations or inequalities of second order, J. Math. Pures Appl. 36 (1957), 235-249.

$4^{-}$Eastham, M.S.P., On the absence of square integrable solutions of the SturmLiouville equation, Lecture Notes in Math. 564, Springer (1976), 72-77.

[5- Eastham, M.S.P. and Kalf, H., Schrödinger-Type Operators with Continuous Spectra. Research Notes in Mathematics 65, Pitman, 1983.

-6- Jansen, K.H. and Kalf, H., On positive eigenvalues of one-body Schrödinger operators: remarks on papers by Agmon and Simon, Comm. Pure Appl. Math, 28 (1975), $747-752$.

[7 - Kato, T., Fundamental properties of Hamiltonian operators of Schrödinger type, Trans. Amer. Math. Soc. 70 (1951), 195-211.

$\left[8_{-}^{-} \longrightarrow\right.$, Growth properties of solutions of the reduced wave equation with a variable coefficient, Comm. Pure Appl. Math. 12 (1959), 403-425.

[9- Odeh, F., Note on differential operators with purely continuous spectrum, Proc. Amer. Math. Soc. 16 (1965), 363-366.

[10- Reed, M. and Simon, B., Analysis of Operators, Academic Press, 1978.

$\left[11_{-}{ }_{-}\right.$Simon, B., On positive eigenvalues of one-body Schrödinger operators, Comm. Pure Appl. Math. 22 (1967), 531-538.

[12- von Neumann, J. and Wigner, E.P., Über merkwürdige diskrete Eigenwerte, $Z$. Phy. 30 (1929), 465-467. 
